\begin{tabular}{|c|l|}
\hline Title & Hierarchy of reaction dynamics in a thermally fluctuating environment \\
\hline Author(s) & Kawai, Shinnosuke; Komatsuzaki, Tamiki \\
\hline Citation & $\begin{array}{l}\text { Physical Chemistry Chemical Physics, 12(27), 7626-7635 } \\
\text { https://doi.org/10.1039/0922080a }\end{array}$ \\
\hline Issue Date & 2010-07-21 \\
\hline Doc URL & http://hdl.handle.net/2115/45145 \\
\hline Rights & Phys. Chem. Chem. Phys., 2010, 12, 7626-7635- Reproduced by permission of the PCCP Owner Societies \\
\hline Type & article (author version) \\
\hline File Information & PCCP12-27_7626-7635.pdf \\
\hline
\end{tabular}

Instructions for use 


\title{
Hierarchy of Reaction Dynamics in a Thermally Fluctuating Environment
}

\author{
Shinnosuke Kawai ${ }^{1 *}$ and Tamiki Komatsuzaki ${ }^{1,2}$ \\ ${ }^{1}$ Molecule \& Life Nonlinear Sciences Laboratory, \\ Research Institute for Electronic Science, \\ Hokkaido University, Kita 20 Nishi 10, \\ Kita-ku, Sapporo 001-0020, Japan \\ and \\ ${ }^{2}$ Core Research for Evolutional Science and Technology (CREST), \\ Japan Science and Technology Agency (JST), \\ Kawaguchi, Saitama 332-0012, Japan \\ * Research Fellow of the Japan Society for the Promotion of Science
}

A boundary to divide the phase space into the reactive and the nonreactive trajectories robustly exists even in thermal fluctuation.

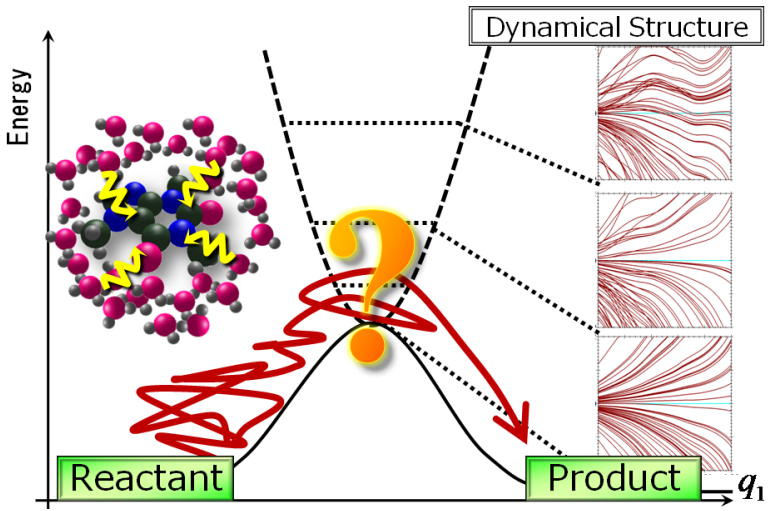

Nonlinear dynamics in the passage over rank-one saddle is investigated as a function of temperature in the presence of stochastic, thermal fluctuation. The analyses are based on a framework we developed recently adopting multidimensional underdamped Langevin equation (without any assumption for the form of the potential of mean force). The framework can in principle provide a single coordinate to enable us to predict the final destination of the reaction in a thermally fluctuating media. At each temperature, the preciseness or the error of the reaction coordinate is evaluated in capturing the true reaction dynamics at different levels of approximations. By using the Müller-Brown potential as an illustrative example, it is found that a hierarchy of dynamical structure exists in the region of rank-one saddle, in which the crossing dynamics qualitatively changes as the temperature increases. We discuss the mechanism of how the reaction coordinate persists, which provides a boundary of the reaction to divide the phase space into the reactive and the nonreactive regions, even in the presence of thermal fluctuation.

\section{INTRODUCTION}

Chemical reactions are ubiquitous in nature, and can be re-

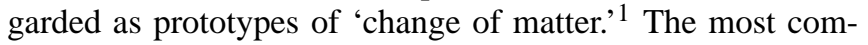
mon class of chemical reactions is a system moving on a single effective potential surface from one local minimum to another, through a saddle region. Chemists have long envisioned the existence of a dividing surface in the saddle region through which the system passes only once in going from one stable state to another. The surface is called the 'transition state (TS). ${ }^{2-9}$ The TS is supposed to lie transverse to a onedimensional coordinate axis, called the 'reaction coordinate,' which describe the progress of the reaction. The concept of the TS has provided us with great insights for understanding not only the rate of chemical reactions but also, for example, ionization of a hydrogen atom in crossed electric and magnetic fields, ${ }^{10}$ isomerization of clusters, ${ }^{11}$ the escape of asteroids from Mars, ${ }^{12}$ and the folding/unfolding of proteins. ${ }^{13}$

Lots of reactions in biology or chemical synthesis occur in condensed phase. The most striking difference from gas phase is that the system is always subject to external stochastic forces by the environment. Even for a fixed initial condition of all the variables in the system, the final destination is not necessarily unique but has a certain probability distribution. Thus, concepts like the resonance overlap, ${ }^{14}$ based on the Hamiltonian formalism, or the deterministic area-preserving Poincaré map ${ }^{15}$ are not feasible for systems with dissipation and stochastic, external driving force that fluctuates thermally. Two approaches to define an appropriate "TS" and/or to overcome the recrossing problem in a fluctuating media have been made by variational TS theory (VTST) ${ }^{8,9}$ which optimizes a configurational dividing surface by minimizing the number of recrossings and by Kramers ${ }^{16}$ and later by Grote and Hynes, ${ }^{17}$ based on (generalized) Langevin formalism which regards the recrossings "as the natural consequence arising from friction by the surrounding environment."

In the former, the VTST has been applied with explicit harmonic bath modes. ${ }^{18-20}$ VTST has provided excellent physical insights about the location of the dividing surface as well as improved expressions for rate constants. In practice, VTST needs a certain small number of selected variables 
to parametrize the dividing surface (usually position coordinate(s) of the system (e.g., solute)). However, as indicated by Van der Zwan and Hynes ${ }^{21}$ and later by Pollak ${ }^{22}$ using harmonic bath Hamiltonian system, the reaction coordinate must be, in principle, a nonlinear function of all the position coordinates and velocities of the reacting system and all the effects exerted by the surroundings. It is non-trivial to identify which degrees of freedom are actually required to be involved. In the latter, the system-bath Hamiltonian approach can formally bridge the descriptions of any Hamiltonian system and the generalized Langevin formulation ${ }^{21,23,24}$ projected onto an arbitrarily chosen coordinate. The very question of the description of dynamical systems in the presence of thermal fluctuation is one of the most intriguing subjects in nonequilibrium statistical mechanics. ${ }^{25,26}$ There remains the fundamental question of what reaction coordinate a system actually follows under the disturbance of thermal fluctuation.

In this article we use the term 'transition state (TS)' to mean the original definition by Wigner, ${ }^{3}$ that is, a dividing surface free from recrossing problem and 'reaction coordinate' a single coordinate that is decoupled from the other coordinate at least in the region of saddle which supports the concept of TS and enables us to predict the destination of the reaction at any instant.

Recently, extensive analyses of nonlinear dynamics around rank-one saddle for gas phase reactions (i.e., Hamiltonian systems) have been performed in terms of geometrical structures of the phase space, ${ }^{10,11,27-43}$ following several developments in experiments ${ }^{44,45}$ and theories ${ }^{46-55}$ on characterizing the regularity in dynamics through saddles. It was revealed that such a TS and a reaction coordinate robustly exist in the multidimensional phase space for a wide class of Hamiltonian systems in the region of rank-one saddle. It was also revealed ${ }^{11,40-43,56,57}$ that a hierarchy of the dynamical structure exists in those regions, dependent on the total energy of the system. In particular the firm mathematical foundation was established for the robust existence of no-return TS, which arises from the generic fact that any resonance cannot occur between reactive and non-reactive degrees of freedom whose frequencies are real and imaginary, respectively, in the region of rank-one saddle. ${ }^{58}$

However, most of reaction system in chemistry and biology is subject to stochastic random force and frictions. This is essentially different from the isolated systems. Recently, we have presented a framework ${ }^{59,60}$ to extract a reaction coordinate that is decoupled from the other coordinates even in a thermally fluctuating environment. The crux was the application of the non-Hamiltonian normal form (NF) theory ${ }^{61}$ together with the time-dependent formulation given by Ref. 62 to multidimensional underdamped Langevin equation with arbitrary potential of mean force. The new reaction coordinate is represented by an analytical functional with respect to the original position coordinates and velocities of the system and the random force and the friction constants exerted by the environment. Similarly to the isolated systems, ${ }^{10,11,27-43}$ under certain moderate conditions, this theory can provide a single coordinate decoupled from the other coordinates which can tell us the final destination of the reaction. In the present pa- per, using the Müller-Brown potential as an illustrative example, we investigate how the crossing dynamics through the first-rank saddle qualitatively change along the reaction coordinate with temperature, and we discuss the hierarchical structure of dynamics and its implication in relevance to reaction control and robustness of functions in biological (nonlinear) systems under thermal fluctuation.

\section{THEORY}

We present a brief summary of the theory we developed in the previous paper. ${ }^{59}$ The framework utilizes an $n$ dimensional Langevin equation:

$$
\ddot{q}=-\frac{\partial U}{\partial q}-\gamma \dot{q}+\xi(t)
$$

where $q=\left(q_{1}, \ldots, q_{n}\right)^{\mathrm{T}}$ is the normal mode position coordinates of the system (solute), $U$ the potential of the mean force, $\gamma$ the friction constant, and $\xi(t)$ describes the random force from the solvent. As an illustrative example, we use the Müller-Brown potential ${ }^{63}$ (See Sec. III) as $U$. In this example, the dimension $n$ is two, but our theory presented in this paper is applicable to systems of any dimension. We use uniform friction $\gamma$ in the numerical example of the present paper, but the theory is also applicable to the case where the friction has off-diagonal part. ${ }^{59}$ The trajectory calculation is performed ${ }^{64}$ with the random force sampled according to the fluctuationdissipation theorem

$$
\left\langle\xi(t) \xi(0)^{\mathrm{T}}\right\rangle=2 k_{\mathrm{B}} T \gamma \delta(t),
$$

where $\xi(0)^{\mathrm{T}}$ is the transpose of the vector $\xi(0), T$ the temperature and $k_{\mathrm{B}}$ Boltzmann constant. The force from the potential $U$ can be expanded in a Taylor series

$$
-\frac{\partial U}{\partial q_{j}}=-k_{j} q_{j}+\sum_{|m| \geq 2} \alpha_{j, m} q_{1}^{m_{1}} \cdots q_{n}^{m_{n}}
$$

with the expansion coefficients $k_{j}$ and $\alpha_{j, m}$ for the linear and the nonlinear parts, respectively. Here, $|m|=\sum_{j} m_{j}$ and $\sum_{|m| \geq 2}$ sums over combinations of $m_{j}$ satisfying $|m| \geq 2$.

Diagonalization of the linear part:

We introduce normal mode coordinates $\left(u_{1}, u_{2}\right)$ in the position-velocity space that diagonalize the linear part

$$
\frac{d}{d t}\left(\begin{array}{c}
q \\
\dot{q}
\end{array}\right)=\left(\begin{array}{cc}
0 & 1 \\
K & -\Gamma
\end{array}\right)\left(\begin{array}{l}
q \\
\dot{q}
\end{array}\right)
$$

where 1 is $n \times n$ unit matrix, $\Gamma$ is equal to $\gamma 1$, and $K$ is the Hessian matrix of $U$ at $q=0$. The normal mode coordinate $u=\left(u_{1}, u_{2}, \ldots, u_{2 n}\right)^{\mathrm{T}}$ is obtained by diagonalizing the matrix

$$
\begin{gathered}
V^{-1}\left(\begin{array}{cc}
0 & 1 \\
K & -\Gamma
\end{array}\right) V=\operatorname{diag}\left(\lambda_{1}, \cdots, \lambda_{2 n}\right), \\
\left(\begin{array}{c}
q \\
\dot{q}
\end{array}\right)=V u
\end{gathered}
$$


Here we assume that the system has a rank-one saddle point to link the reactant and the product regions such that $\lambda_{1}>0$, $\operatorname{Re}\left(\lambda_{j}\right)<0(j=2, \ldots, 2 n)$. The rank-one saddle point includes both the information of the linear parts of potential of mean force (i.e., the curvature of the potential) and friction constants. The $\lambda_{1}>0$ corresponds to the unstable motion sliding down the barrier. The analytical expressions of the eigenvalues $\lambda$ is given in Appendix of Ref. 59.

After the diagonalization, the equation of motion becomes

$$
\frac{d}{d t} u_{j}=\lambda_{j} u_{j}+\tilde{\xi}_{j}(t)+\sum_{|m| \geq 2} \beta_{j, m} u_{1}^{m_{1}} u_{2}^{m_{2}} \cdots u_{2 n}^{m_{2 n}},
$$

where $\tilde{\xi}(t)=V\left(\begin{array}{c}0 \\ \xi(t)\end{array}\right)$, and the coefficients $\beta_{j, m}$ are obtained by substituting Eq. (5) into the nonlinear terms in Eq. (3). The surface defined by $u_{1}=0$ would perform the role of the boundary between reactive and non-reactive trajectories if there were no random force or nonlinearities. In reality, however, trajectories cross the $u_{1}=0$ axis many times due to the random force and nonlinear couplings.

\section{Shift of the origin:}

Recent studies ${ }^{65-68}$ introduced relative coordinates, which we call $x_{j}$ here, by

$$
u_{j}=S\left[\lambda_{j}, \tilde{\xi}_{j}\right](t)+x_{j}
$$

where we have introduced a notation $S$ as in Refs. ${ }^{62,68}$ :

$$
S\left[\lambda_{j}, \tilde{\xi}_{j}\right](t)= \begin{cases}\int_{-\infty}^{0} \exp \left(-\lambda_{j} \tau\right) \tilde{\xi}_{j}(t+\tau) d \tau & \left(\operatorname{Re} \lambda_{j}<0\right) \\ -\int_{0}^{+\infty} \exp \left(-\lambda_{j} \tau\right) \tilde{\xi}_{j}(t+\tau) d \tau & \left(\operatorname{Re} \lambda_{j}>0\right)\end{cases}
$$

Then the equation of motion for $x$ is given by

$$
\frac{d}{d t} x_{j}=\lambda_{j} x_{j}+\sum_{|m| \geq 0} f_{j, m}(t) x_{1}^{m_{1}} x_{2}^{m_{2}} \cdots x_{2 n}^{m_{2 n}},
$$

where the coefficients $f_{j, m}(t)$ are obtained from the nonlinear terms in Eq. (6). The expansion coefficients $f_{j, m}(t)$ are now time-dependent due to the time-dependent shift [Eq. (7)]. If there were no nonlinear terms in the potential $U$, the equation of motion in $x_{1}$ would not depend on the random force. Then the solution would be $x_{1}(t)=x_{1}\left(t_{0}\right) \exp \left[\lambda_{1}\left(t-t_{0}\right)\right]$ where $t_{0}$ is a certain instant of time. Since $\lim _{t \rightarrow+\infty} \exp \left[\lambda_{1}\left(t-t_{0}\right)\right]=\infty$, the system would move away from the barrier region as $t$ increases, with the direction [the sign of $x_{1}(t)$ ] determined solely by the sign of $x_{1}\left(t_{0}\right)$. However, due to the presence of the nonlinear terms, the dynamics of $x_{j}$ depends on all $\tilde{\xi}$ through the time dependence in $f_{j}^{(k)}(x, t)$ that originates from that of random force $\xi(t)$. Hereinafter for the sake of brevity we will not insert the notations of $\xi(t)$ and $\lambda$ into the notations of time-dependent coefficients appeared in the following procedures (i.e., $c_{j}(y, t), w_{j}(y, t)$, and $\left.g_{j}(y, t)\right)$ but all these coefficients generally depend on both $\xi(t)$ and $\lambda$ exerted by the surrounding environment.

Nonlinear transformation to incorporate the effect of nonlinear couplings:
To incorporate the effect of nonlinear terms in Eq. (9), we employ non-Hamiltonian normal form (NF) theory ${ }^{61}$ to introduce a nonlinear transformation from $x$ to $y$ so that the equation of motion for $y_{1}$ contains no coupling with the other coordinates. The calculation proceeds in a similar way to Lie canonical perturbation theory ${ }^{69,70}$ (a classical analog of Van Vleck perturbation theory). The equation of motion in $x$ [Eq. (9)] is cast into

$$
\frac{d}{d t} y_{j}=\lambda_{j} y_{j}+c_{j}(y, t)
$$

by the transformation $x_{j}=y_{j}+w_{j}(y, t)$. Introducing polynomial expansions for two unknown functions $\left(c_{j}\right.$ and $\left.w_{j}\right)$ as

$$
\begin{aligned}
& c_{j}(y, t)=\sum_{m} c_{j, m}(t) y_{1}{ }^{m_{1}} \cdots y_{2 n}{ }^{m_{2 n}}, \\
& w_{j}(y, t)=\sum_{m} w_{j, m}(t) y_{1}{ }^{m_{1}} \cdots y_{2 n}{ }^{m_{2 n}}
\end{aligned}
$$

The coefficients $w_{j, m}(t)$ are determined so as to diminish $c_{j, m}(t)$ order by order: Substituting these unknown functions into Eq. (9), we obtain

$$
\left(\langle\lambda, m\rangle-\lambda_{j}+\frac{d}{d t}\right) w_{j, m}(t)=g_{j, m}(t)-c_{j, m}(t),
$$

Where $\langle\lambda, m\rangle=\sum_{k=1}^{2 n} \lambda_{k} m_{k}$ and $g_{j, m}(t)$ are the coefficients of the polynomial expansion of $g_{j}(y, t) \stackrel{\text { def }}{=} f_{j}(y+w, t)-$ $\sum_{i=1}^{2 n} c_{i}(y, t) \frac{\partial w_{j}(y, t)}{\partial y_{i}}$. As is shown in the previous paper, ${ }^{59}$ in the process of determining $c_{j, m}(t)$ and $w_{j, m}(t)$ perturbationally order by order, $g_{j, m}(t)$ is known from the results of the lower orders. There thus exist two unknown quantities $c_{j, m}(t)$ and $w_{j, m}(t)$ with the known $g_{j, m}(t)$ at each order. One can then determine either of the two unknown quantities as one wishes. To simplify the equation of motion for the new variables $y_{1}$, one can eliminate $c_{1, m}(t)$ order by order by setting

$$
w_{1, m}(t)=S\left[\lambda_{1}-\langle\lambda, m\rangle, g_{1, m}\right](t),
$$

using the $S$-symbol as in Eqs. (7) and (8). ${ }^{62,68}$ For $m_{1}=$ $1, m_{2}=\cdots=m_{2 n}=0$, we have $\lambda_{1}-\langle\lambda, m\rangle=0$, for which case the $S$-symbol cannot be defined. This makes it necessary to incorporate a term $c_{1, m}(t)$ in Eq. (12), where $m_{1}=1, m_{2}=$ $\cdots=m_{2 n}=0$. The equation of motion thus becomes

$$
\frac{d}{d t} y_{1} \approx\left\{\lambda_{1}+c_{1}(t)\right\} y_{1}
$$

with a time-dependent coefficient $c_{1}(t)$. The solution of Eq. (14) is given by

$$
y_{1}(t) \approx y_{1}\left(t_{0}\right) \exp \left[\int_{t_{0}}^{t}\left(\lambda_{1}+c_{1}\left(t^{\prime}\right)\right) d t^{\prime}\right],
$$

where $t_{0}$ is a certain instant of time (the initial condition). The exponent in Eq. (15) goes to infinity as $t \rightarrow+\infty$ if the longtime average of the perturbative term $c_{1}(t)$ is less than $\lambda_{1}$. Since $\lambda_{1}$ is $O(1)$ and $c_{1}(t)$ is $O(\varepsilon)$ with a small parameter $\varepsilon$ in the perturbation calculation, this inequality can usually be expected to hold. As long as this condition is fulfilled, the 
sign of $y_{1}$ at time $t_{0}$ can predict the destination of the reaction a priori before the system reaches either the reactant or the product region.

Once we determine the functions $w_{j}^{(k)}(y, t)$ up to a desired order, the transformation from $y$ to $x$ is given by $x_{j}=y_{j}+$ $w_{j}(y, t)$. The inverse transformation, i.e., $y$ as a function of $x$ (hence of $q$ ), can be given by the iterative technique [Eqs. (27) and $(28)$ in the previous paper $\left.{ }^{59}\right]$. The transformation from the original coordinates $(q, \dot{q})$ (through $u$ and $x$ ) to $y$ can be expressed in the form of a polynomial expansion

$$
\begin{aligned}
y_{1}= & a_{1} q_{1}+a_{2} \dot{q}_{1}-S\left[\lambda_{1}, \tilde{\xi}_{1}\right](t)+F_{0}[\xi](t) \\
& +\sum_{|m| \geq 2} w_{m} q_{1}{ }^{m_{1}} \cdots q_{n}^{m_{n}} \dot{q}_{1}^{m_{n+1}} \cdots \dot{q}_{n}^{m_{2 n}} \\
& +\sum_{|m| \geq 1} F_{m}[\xi](t) q_{1}^{m_{1}} \cdots q_{n}^{m_{n}} \dot{q}_{1}^{m_{n+1}} \cdots \dot{q}_{n}^{m_{2 n}}
\end{aligned}
$$

In the previous paper, ${ }^{59}$ the physical interpretation of each term appearing in Eq. (16) was also given. Briefly, the first two terms correspond to linear approximation. The third term $-S\left[\lambda_{1}, \tilde{\xi}_{1}\right](t)$ expresses direct environment effect, that is, the kick by the random force along the reactive normal mode direction. The terms with the coefficients $w_{m}$ come from the nonlinearity of the system. The rest terms (with $F_{0}[\xi](t)$ and $F_{m}[\xi](t)$ ) are the combined effects of the environment and the nonlinearity, in the sense that they arise only when there exist both the random force and the nonlinearity in the potential of mean force. Note here that all the coefficients depend on the friction constants, while only $S\left[\lambda_{1}, \tilde{\xi}_{1}\right](t), F_{0}[\xi](t)$, and $F_{m}[\xi](t)$ include the random force and are therefore timedependent.

Eq. (16) above can be calculated only when one knew the instance of the random force $\xi(t)$ for all the past and the future time in advance. In practice, however, what one may assume a priori is the statistical properties of $\xi(t)$ as an ensemble rather than a single instance. Therefore the previous paper ${ }^{59}$ also has discussed taking the ensemble of $y_{1}$ averaged over all realizations of $\xi(t)$ :

$$
\begin{aligned}
\left\langle y_{1}\right\rangle= & a_{1} q_{1}+a_{2} \dot{q}_{1}+\bar{F}_{0}\left(k_{\mathrm{B}} T\right) \\
& +\sum_{|m| \geq 2} w_{m} q_{1}{ }^{m_{1}} \cdots q_{n}{ }^{m_{n}} \dot{q}_{1}^{m_{n+1}} \cdots \dot{q}_{n}^{m_{2 n}} \\
& +\sum_{|m| \geq 1} \bar{F}_{m}\left(k_{\mathrm{B}} T\right) q_{1}{ }^{m_{1}} \cdots q_{n}^{m_{n}} \dot{q}_{1}^{m_{n+1}} \cdots \dot{q}_{n}^{m_{2 n}}
\end{aligned}
$$

where the time-independent coefficients $\bar{F}_{0}\left(k_{\mathrm{B}} T\right)$ and $\bar{F}_{m}\left(k_{\mathrm{B}} T\right)$ are the averages of the corresponding terms in Eq. (16) and are functions of temperature through Eq. (2). The direct environment effect vanishes by the average of $\xi(t)$.

\section{A. Two types of normal form}

\section{1. partial normal form}

For the purpose of telling the fate of the reaction, we need only to know the motion along the unstable direction $y_{1}$ which corresponds to the motion sliding down the barrier. Thus we can normalize the equation of $\dot{y}_{1}$ while leaving other modes as they are:

$$
\begin{aligned}
\dot{y}_{1} & =\left[\lambda_{1}+c_{1}(t)\right] y_{1}+O\left(\varepsilon^{N+1}\right) \\
\dot{y}_{2} & =f_{2}(y) \\
\dot{y}_{3} & =f_{3}(y) \\
& \vdots \\
\dot{y}_{2 n} & =f_{2 n}(y) .
\end{aligned}
$$

We call this "partial normal form." The solution for the motion of $y_{1}$ is given by

$$
y_{1}(t)=y_{1}\left(t_{0}\right) \exp \left[\int_{t_{0}}^{t}\left(\lambda_{1}+c_{1}\left(t^{\prime}\right)\right) d t^{\prime}\right]+O\left(\varepsilon^{N+1}\right),
$$

Here, $\lambda_{1}$ comes from the harmonic approximation, which corresponds to the zeroth order of the perturbation in our normal from treatment. ${ }^{59}$ The other term $c_{1}(t)$ originates from the effect of nonlinearity, which begins with first-order perturbation term.

Of particular importance is the behavior in $t \rightarrow+\infty$. The exponent in Eq. (19) goes to infinity as $t \rightarrow+\infty$ if the longtime average of the perturbative terms in $c_{1}(t)$ is less than $\lambda_{1}$

$$
\lim _{t \rightarrow+\infty}\left|\frac{1}{t} \int_{t_{0}}^{t} c_{1}\left(t^{\prime}\right) d t^{\prime}\right|<\lambda_{1} .
$$

Since the left-hand side is of first order of perturbation and the right-hand side is zeroth order, this inequality can usually be expected to hold. As long as this condition is fulfilled, we have

$$
\lim _{t \rightarrow+\infty} y_{1}(t)= \begin{cases}+\infty & \left(y_{1}\left(t_{0}\right)>0\right) \\ -\infty & \left(y_{1}\left(t_{0}\right)<0\right)\end{cases}
$$

The increase of $\left|y_{1}(t)\right|$ means that the system departs from the vicinity of the saddle point, going either to the products or to the reactants. The direction of this departing motion is determined by the sign of $y_{1}$. The most important consequence is that, irrespective of the other modes $\left(y_{2}, \ldots, y_{2 n}\right)$, only the knowledge of the sign of $y_{1}$ at any one moment in the region of saddle is sufficient to tell the fate of the reaction.

Figure 1 shows a schematic picture of the phase space flow projected onto the $y_{1}-y_{2}$ plane. The trajectories are drawn for the case of two independent linear equations

$$
\begin{aligned}
& \dot{y_{1}}=\lambda_{1} y_{1}, \\
& \dot{y_{2}}=\lambda_{2} y_{2} .
\end{aligned}
$$

We can think of the left half of the plane as corresponding to the reactant and the right half the product. It can be seen that the trajectories with $y_{1}>0$ goes from the reactant to the product or vice versa, while those with $y_{1}<0$ are reflected back by the barrier. This figure has a good correspondence with the preceding studies on the Hamiltonian systems ${ }^{10,11,27-43}$ where the canonical conjugate pair $\left(\bar{q}_{1}, \bar{p}_{1}\right)$ was used as the "reaction coordinate." In the present case, however, the equation of motion of $y_{1}$ depends also on time $t$, and that of $y_{2}$ on all the 


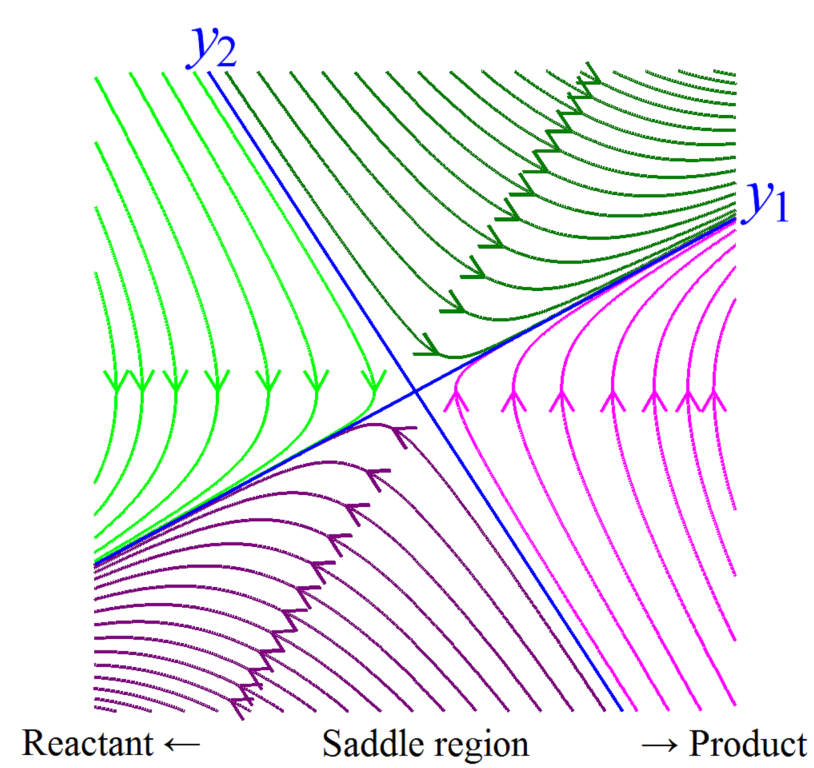

FIG. 1: A schematic picture of the phase space flow projected onto the $y_{1}-y_{2}$ plane. It is seen that the trajectories with positive $y_{1}$ goes from the left side (the reactant) to the right side (the product), or vice versa, while those with negative $y_{1}$ are nonreactive. Note that the sign of $y_{1}$ is invariant, and therefore the knowledge of the sign of $y_{1}$ at any instant time is sufficient to predict the fate of the reaction. The set $y_{1}=0$ makes an invariant set, which functions as a boundary of the reaction.

other coordinates [Eq. (18)], thus plotting only $y_{1}$ and $y_{2}$ is not a correct way for the representation of the phase space. Since the sign of $y_{1}$ is sufficient for the purpose of knowing the fate of the reaction, we will in the present paper plot the phase space flow in the $\left(t, y_{1}\right)$-plane, where the flow can be analyzed independently from the other coordinates in the case of the partial NF [Eq. (18)].

For the purpose of illustrating the transformation, we plot in Fig. 2 some representative trajectories generated by a numerical simulation of the Langevin equation in the three different coordinates, $u_{1}, x_{1}$ and $y_{1}$. Computational details of the simulation is given in Sec. III. Fig. 2(a) plots the time evolution of the normal mode coordinate $u_{1}$ [defined in Eq. (5)] along each trajectory. The plots show noisy shapes due to the random force. In Fig. 2(b), they have become much smoother in the shifted coordinate [Eq. (7)], which means that the shift can cancel the effect of the random force to some extent. However, for the purpose of distinguishing the reactive and the nonreactive trajectories, we cannot use the line $x_{1}=0$ to divide them because some trajectories cross it. The crossings are due to the effect of nonlinearity. The normal form transformation from $x$ to $y$ treats the nonlinear part. The plot with time $t$ and the NF reaction coordinate $y_{1}$ is plotted in Fig. 2(c). It is seen that the line $y_{1}=0$ separates the reactive and the nonreactive trajectories [note that a large positive (negative) value of $y_{1}$ corresponds to the product (reactant), as in Fig. 1]. As one can see in Sec. IV, as temperature increases, $y_{1}=0$ in the partial NF can no longer act as a reaction boundary to distinguish the reactive and the nonreactive trajectories.

\section{2. minimal normal form}

One of the main theme of this article is to propose another normal form which has not been noticed in the literature insofar, resulting in the robust reaction boundary to divide the space into reactive and nonreactive trajectories even though the reaction coordinate $y_{1}$ is no longer decoupled from the other in the partial NF structure. This normal form termed as minimal NF is designed to reduce the extent of normalization as follows.

$$
\begin{aligned}
\dot{y}_{1} & =\left[\lambda_{1}+\tilde{c}_{1}(y, t)\right] y_{1}+O\left(\varepsilon^{N+1}\right) \\
\dot{y}_{2} & =f_{2}(y) \\
\dot{y}_{3} & =f_{3}(y) \\
& \vdots \\
\dot{y}_{2 n} & =f_{2 n}(y),
\end{aligned}
$$

where $\tilde{c}_{1}$ can now be a function of $y$. In other words, we eliminate the terms with $m_{1}=0$ from the equation of $\dot{y}_{1}$, but retain all the terms with $m_{1} \geq 1$ (that is, no function consisting only of $y_{2}, y_{3}, \ldots$ in the right hand side of equation of $\dot{y}_{1}$ ). The solution is formally given by

$$
y_{1}(t)=y_{1}\left(t_{0}\right) \exp \left[\int_{t_{0}}^{t}\left(\lambda_{1}+\tilde{c}_{1}\left(y\left(t^{\prime}\right), t^{\prime}\right)\right) d t^{\prime}\right]
$$

This solution is only formal in the sense that the right hand side contains $y\left(t^{\prime}\right)$ which would have to be solved according to the very equation of motion Eq. (23). However, the important point is that $y_{1}=0$ forms an invariant set just as in the case of partial normal form (i.e., $d y_{1} / d t=0$ if $y_{1}=0$ ). This implies that once the system is in the manifold of $y_{1}=0$, the system should remain to reside there for $t \rightarrow \pm \infty$. In other words, no trajectory can cross the manifold $y_{1}=0$ from the region $y_{1}>0$ to $y_{1}<0$, or vice versa. Consequently, once we know the sign of $y_{1}\left(t_{0}\right)$ at any instant $t_{0}$ in the saddle region, it fixes the sign of $y_{1}$ for $t \rightarrow \infty$, telling us the final destination of the reaction (the reactant or the product regions, see Fig. 1). The condition corresponding to Eq. (20) is given by

$$
\lim _{t \rightarrow+\infty}\left|\frac{1}{t} \int_{t_{0}}^{t} \sum_{k=1}^{N} \varepsilon^{k} \tilde{c}_{1}^{(k)}\left(y\left(t^{\prime}\right), t^{\prime}\right) d t^{\prime}\right|<\lambda_{1} .
$$

This is more difficult to check because it contains the solution $y\left(t^{\prime}\right)$ to the equation of motion. However, this can usually be expected to hold since the right hand side is of first order of perturbation, while the left hand side is of zeroth order.

The minimal normal form is expected to have better convergence property because it contains less terms in the transformation. The difference of convergence will be discussed in the following sections. 

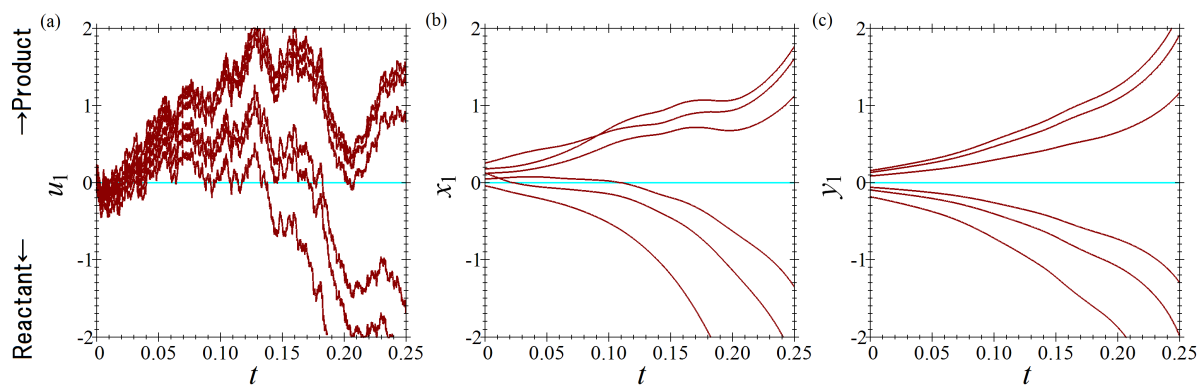

FIG. 2: Some representative trajectories for $k_{\mathrm{B}} T=2$ plotted in time $t$ and (a) phase space normal mode coordinate $u_{1}$, which is a linear combination of the position coordinate $q_{1}$ and the velocity $\dot{q}_{1}$, (b) shifted coordinate $x_{1}$ with the shift depending on time to incorporate the effect of the random force (in the linear part), and (c) normal form coordinate $y_{1}$ constructed by a nonlinear transformation from $x$. Computational details is given in Sec. III. The trajectories have complicated shapes due to the random force in (a), which is attenuated in (b) by the time-dependent shift. The trajectories plotted in (b) still experiences crossings with each other and crossings with the line $x_{1}=0$. If the coordinate $y_{1}$ is used, there is no crossings and the line $y_{1}=0$ separates the reactive and the nonreactive trajectories.

\section{B. Error estimate}

The present theory is based on series expansions and truncations. The coordinate transformation and the equation of motion for $y$ are only an approximate description. It must be checked whether the error of this approximation is sufficiently small. For Hamiltonian systems, an energy error criterion was used $^{38,39}$ to check the convergence property of the NF transformation. This exploits the fact that the time-independent canonical transformation (if exact) leaves the value of the Hamiltonian unchanged. In the present case, however, the system is non-Hamiltonian and we cannot use total energy as a criterion for convergence. We define the following criterion for the error check in our dissipative system:

$$
\begin{aligned}
\delta \dot{y}_{1} \stackrel{\text { def }}{=} & \frac{d}{d t} y_{1}(q(t), \dot{q}(t))- \\
& \left\{\lambda_{1}+\tilde{c}_{1}(y(q(t), \dot{q}(t), t)\} y_{1}(q(t), \dot{q}(t)),\right.
\end{aligned}
$$

which is the error in the velocity along $y_{1}$. In the above equation, $q(t)$ and $\dot{q}(t)$ are calculated by numerical simulation and then substituted into the transformation of $y_{1}$ with given $\xi(t)$ and $\gamma$ by Eq. (16). The first term on the right hand side is thus the numerical derivative of $y_{1}$ with respect to time $t$. The rest terms correspond to the right hand side of the equation of motion for $y_{1}$ [Eq. (23)]. If the transformation and the equation of motion were exact, it should be equal to the first term on the right hand side of Eq. (26). Hence the quantity $\delta \dot{y}_{1}$ can be regarded as an estimate of the error in the NF transformation.

In order to compare the error or preciseness at the different levels of approximations, we take the ensemble average of the square of $\delta \dot{y}_{1}$ relative to the average of the square of $\dot{y}_{1}$ in the region of the saddle:

$$
\frac{\left\langle\left(\delta \dot{y}_{1}\right)^{2}\right\rangle}{\left\langle\left(\dot{y}_{1}\right)^{2}\right\rangle}
$$

where the bracket denotes thermal average on the surface:

$$
\langle A\rangle \stackrel{\text { def }}{=} \int d q d \dot{q} A(q, \dot{q}) \delta\left(s(q)-s_{0}\right) \exp \left[-\frac{E(q, \dot{q})}{k_{\mathrm{B}} T}\right] .
$$

Here $A$ is any function of $(q, \dot{q})$, and $E$ is the energy defined as the kinetic energy $\left(\dot{q}_{1}^{2}+\dot{q}_{2}^{2}\right) / 2$ plus the potential $U$. The condition $s(q)=s_{0}$ gives a surface over which the averages are taken. In this paper we take the surface as the ridge of the potential surface (see Sec. III and Fig. 3), which can be considered as lying approximately between the reactant and the product.

\section{MODEL}

As a model system we use Müller-Brown potential, ${ }^{63}$ which has often been used as a test system for searching algorithms of minima and saddle points. The potential $U$ is expressed in terms of two position coordinates $\left(Q_{1}, Q_{2}\right)$ by

$$
\begin{aligned}
U\left(Q_{1}, Q_{2}\right)= & \sum_{i=1}^{4} A_{i} \exp \left[a_{i}\left(Q_{1}-Q_{1, i}^{0}\right)^{2}\right. \\
& \left.+b_{i}\left(Q_{1}-Q_{1, i}^{0}\right)\left(Q_{2}-Q_{2, i}^{0}\right)+c_{i}\left(Q_{2}-Q_{2, i}^{0}\right)^{2}\right],
\end{aligned}
$$

with $A=\left(A_{1}, A_{2}, A_{3}, A_{4}\right)=(-200,-100,-170,15)$, $a=(-1,-1,-6.5,0.7), \quad b=(0,0,11,0.6), \quad c=$ $(-10,-10,-6.5,0.7), \quad Q_{1}^{0}=(1,0,-0.5,-1), \quad$ and $Q_{2}^{0}=(0,0.5,1.5,1)$. The contour plot of the potential is shown in Fig. 3. This system possesses three minima and two saddle points. In this paper we deal with the saddle point with higher energy (called "saddle 2" in preceding papers), which has a significant asymmetrical feature of potential compared to the other saddle. The barrier height from each minimum is 41 and 107 (in the unit of energy), respectively (the barrier height of the other "saddle 1" at lower energy is 9 and 36, respectively). ${ }^{59,60}$ The potential and the directions of the normal modes in the configuration space are shown in Fig. 3. The orange curve in the figure shows the ridge of the potential surface, on which the ensemble is taken in Eq. (28).

The friction constant is set to be proportional to unit matrix $\gamma_{i j}=30 \delta_{i j}$. This value was regarded as the underdamped case because it is of the same order with the normal mode frequency of the system at the saddle point. In this paper, NF 


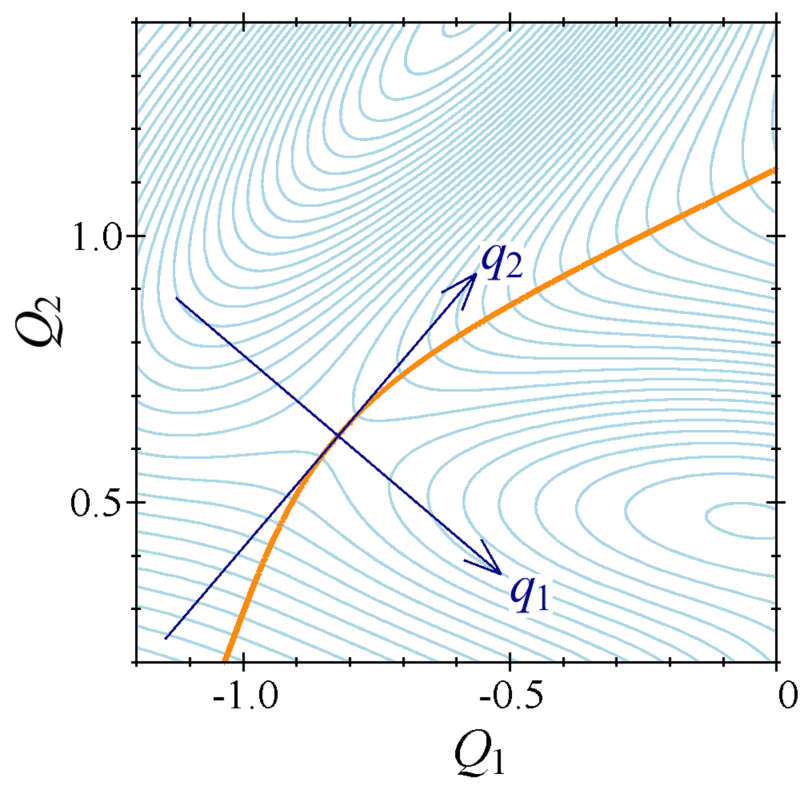

FIG. 3: Configuration space normal mode coordinates at saddle 2 superimposed with the contours of the potential surface spaced by 1 . The coordinate $q_{1}$ corresponds to the reactive direction, and $q_{2}$ the non-reactive one. The ridge of the potential is shown by bold orange line.

calculations are performed up to the second order of perturbation.

To quantify the validity of Eqs. (9), (18), and (23) for describing the dynamics of the system in a fluctuating media, we investigate the flows of trajectories in the position-velocity space (also called phase space in this article). We fix $a$ random force $\xi(t)$ made from one sequence of random numbers at each temperature. Eqs. (9), (18), and (23) themselves are formulated for any $\xi(t)$. The reason for fixing the random force $\xi(t)$ in the analyses is that, once the random force $\xi(t)$ is fixed, one can scrutinize the uniqueness of the solution of the dynamical systems of $x_{i}[q, \dot{q} ; \xi], y_{i}[q, \dot{q} ; \xi]$ along the original Langevin dynamics $(q, \dot{q} ; \xi(t))$ obeying Eq. (1). One can then evaluate the validity of the representation of Eqs. (9), (18), and (23) as a function of temperature. We employ the long period random number generator of L'Ecuyer with BaysDurham shuffle. ${ }^{71}$

\section{RESULTS AND DISCUSSION}

\section{A. The preciseness of NF approximations}

Figure 4 shows the error of the velocity along $y_{1}$ calculated for harmonic approximation $\left(\frac{d}{d t} x_{1} \approx \lambda_{1} x_{1}\right.$, see Eq. 9), the partial NF [Eq. (18)] and the minimal NF [Eq. (23)]. The errors increase with temperature for all the three cases. This is because the system can experience a wider region of the potential surface around the saddle point, thus, of a larger nonlinearity, as temperature increases. Although the error of the har-

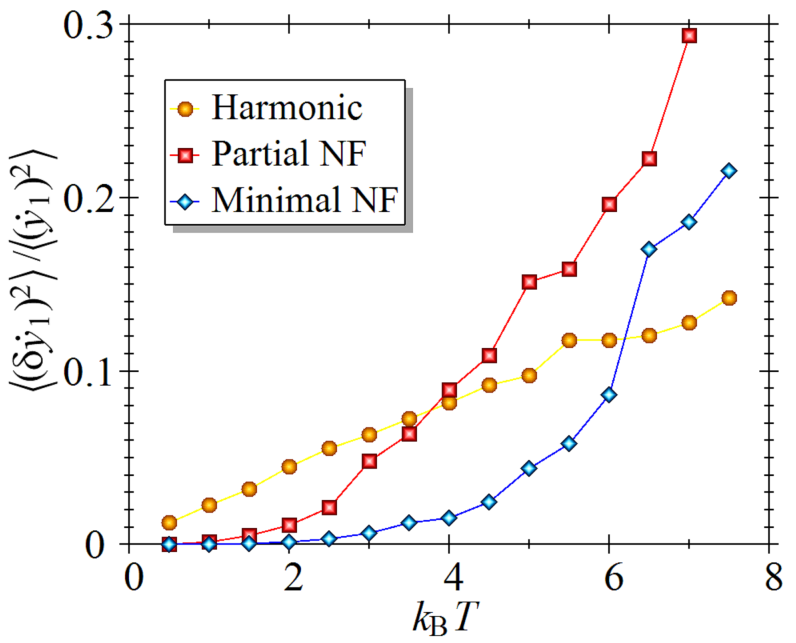

FIG. 4: Error estimates for the NF approximation to the true dynamics as functions of the temperature. Circle, square, and diamond correspond to harmonic approximation, partial NF, and minimal NF, respectively.

monic approximation starts to exceed a few percents at even $k_{\mathrm{B}} T \approx 1$, the error of the partial NF remain less than a few percents up to $k_{\mathrm{B}} T \approx 2$. The minimal NF gives yet better approximation with the error being only a few percents up to $k_{\mathrm{B}} T \approx 4$.

\section{B. Geometry of phase space flow}

To explore how the dynamical structures of the system change in the presence of thermal fluctuation with temperature, we investigate the flows of trajectories in the positionvelocity space. For each plot, we use one instance of the random force $\xi(t)$ made from an arbitrary chosen, single same sequence of random numbers and pick sixty initial conditions randomly sampled on the ridge of the potential surface. Then we propagate the trajectories for these initial conditions and plot them in terms of time $t$ and one coordinate $\left(x_{1}\right.$ or $\left.y_{1}\right)$. Figure 5 shows the flow of trajectories in the $t-x_{1}$ space at the temperature $k_{\mathrm{B}} T=0.1$. At such low temperature, the harmonic part of the potential makes a good approximation of the system, so that the equation of motion is almost given by [see Eq. (9)]

$$
\frac{d}{d t} x_{1}=\lambda_{1} x_{1}
$$

which has a solution

$$
x_{1}(t)=x_{1}\left(t_{0}\right) \exp \left(\lambda_{1}\left(t-t_{0}\right)\right)
$$

where $t_{0}$ is an instantaneous time (initial condition) while the system is in the region of saddle. Thus, for each trajectory, the absolute value of $x_{1}$ increases monotonically with $t$. All the trajectories are repelled from the line $x_{1}=0$, which thus functions as the "boundary" between the reactive and the nonreactive trajectories. No trajectory can cross this boundary. 
In other words, the line $x_{1}=0$ makes an invariant set of the system. Note also that no two trajectories cross each other. This is due to the uniqueness of the solution to the differential equation (30), which can be (at this temperature) regarded as a good approximation of the true dynamics. That is, the same initial condition $x_{1}\left(t_{0}\right)$ will lead to the same solution.

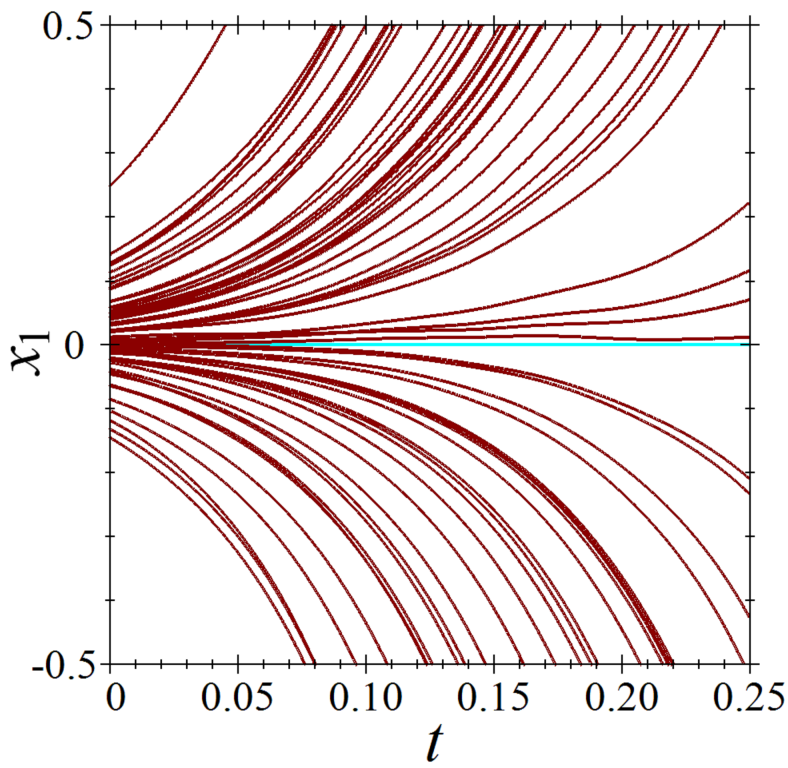

FIG. 5: Trajectories plotted in time $t$ and the shifted normal mode coordinate $x_{1}$ at $k_{\mathrm{B}} T=0.1$. A light blue line marks the set $x_{1}=0$, which should functions as the boundary of reaction if the harmonic approximation is valid for this system. Note that a clear lamination of the flow and no crossing with $x_{1}=0$ are observed.

On the other hand, Fig. 6 (a) shows the plot of trajectories in the $t-x_{1}$ space at higher temperature of $k_{\mathrm{B}} T=2$. Two remarkable changes from the case of $k_{\mathrm{B}} T=0.1$ are observed: (1) Some trajectories cross the line $x_{1}=0$. (2) There are crossings of two trajectories with each other. These facts implies that the harmonic approximation [Eq. (30)] does not give the correct picture of the dynamics any more. The crossings of the trajectories with each other are understood from the effect of couplings as follows. Given one instance of the random force $\xi(t)$, the solution should be unique once we specify the initial condition for the whole coordinate $\left(x_{1}\left(t_{0}\right), x_{2}\left(t_{0}\right), x_{3}\left(t_{0}\right), x_{4}\left(t_{0}\right)\right)$ at time $t=t_{0}$ (irrespective of the validity of harmonic approximation) [See Eq. (9)]. However, the figure is a projection onto the $x_{1}$ axis. Some trajectories can have the same value of $x_{1}\left(t_{0}\right)$ but different values $x_{2}\left(t_{0}\right), x_{3}\left(t_{0}\right), x_{4}\left(t_{0}\right)$. Such trajectories occupy the same point in the $t-x_{1}$ space at $t=t_{0}$ but have different time evolution due to the coupling between $x_{1}$ and the other coordinates $\left(x_{2}, x_{3}, x_{4}\right)$. For harmonic regions the couplings are negligible, which is the reason why we did not observe the crossings at $k_{\mathrm{B}} T=0.1$ (Fig. 5).

Fig. 6 (b) plots the same trajectories in Fig. 6(a) in the $t-y_{1}$ space where $y_{1}$ is obtained by partial NF (Sec. II A 1 ). Hereafter we denote this coordinate as $y_{1}^{\mathrm{p}}$ to distinguish from the
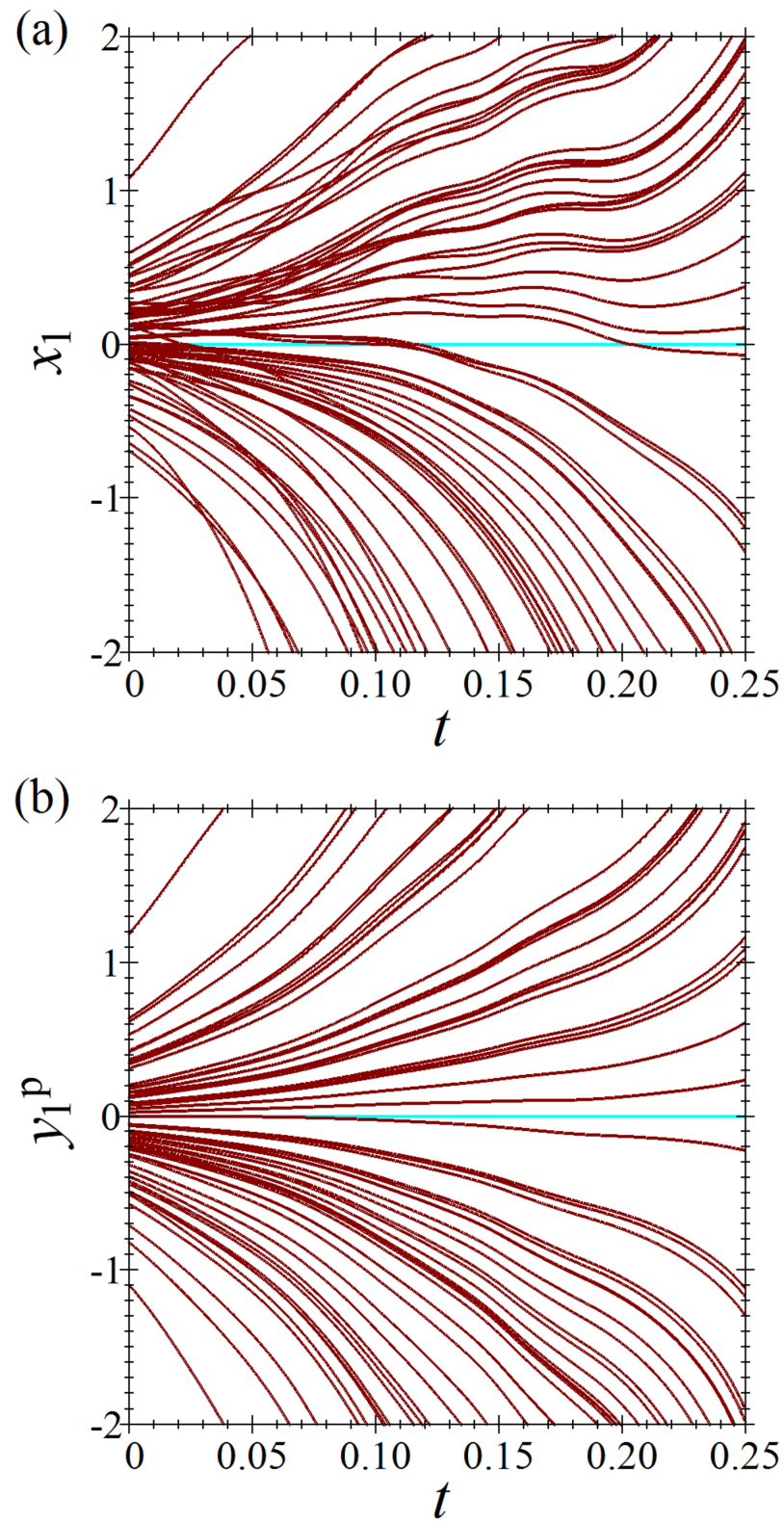

FIG. 6: Trajectories at the temperature $k_{\mathrm{B}} T=2$, plotted in (a) time $t$ and the shifted normal mode coordinate $x_{1}$, and (b) time $t$ and the partial normal form coordinate $y_{1}$. Light blue lines mark the set $x_{1}=$ 0 and $y_{1}=0$.

minimal NF (Sec. II A 2). In the partial NF framework, neither crossings through $y_{1}^{\mathrm{p}}=0$ nor crossings between trajectories are observed, and the phase space flow is tidily laminated. That is, the solution is uniquely determined with the same value of $y_{1}^{\mathrm{p}}\left(t_{0}\right)$ without mattering the values of $y_{2}, y_{3}$, and $y_{4}$. This implies that the normal form reaction coordinate $y_{1}^{\mathrm{p}}$ is actually decoupled from the other coordinates. Note also that there is an invariant set defined by $y_{1}^{\mathrm{p}}=0$ to separate the reactive and the non-reactive trajectories (at least) up to the order of perturbation.

As can be seen from Fig. 4, the partial NF also starts to suffer from larger errors at higher temperature whilst the mini- 

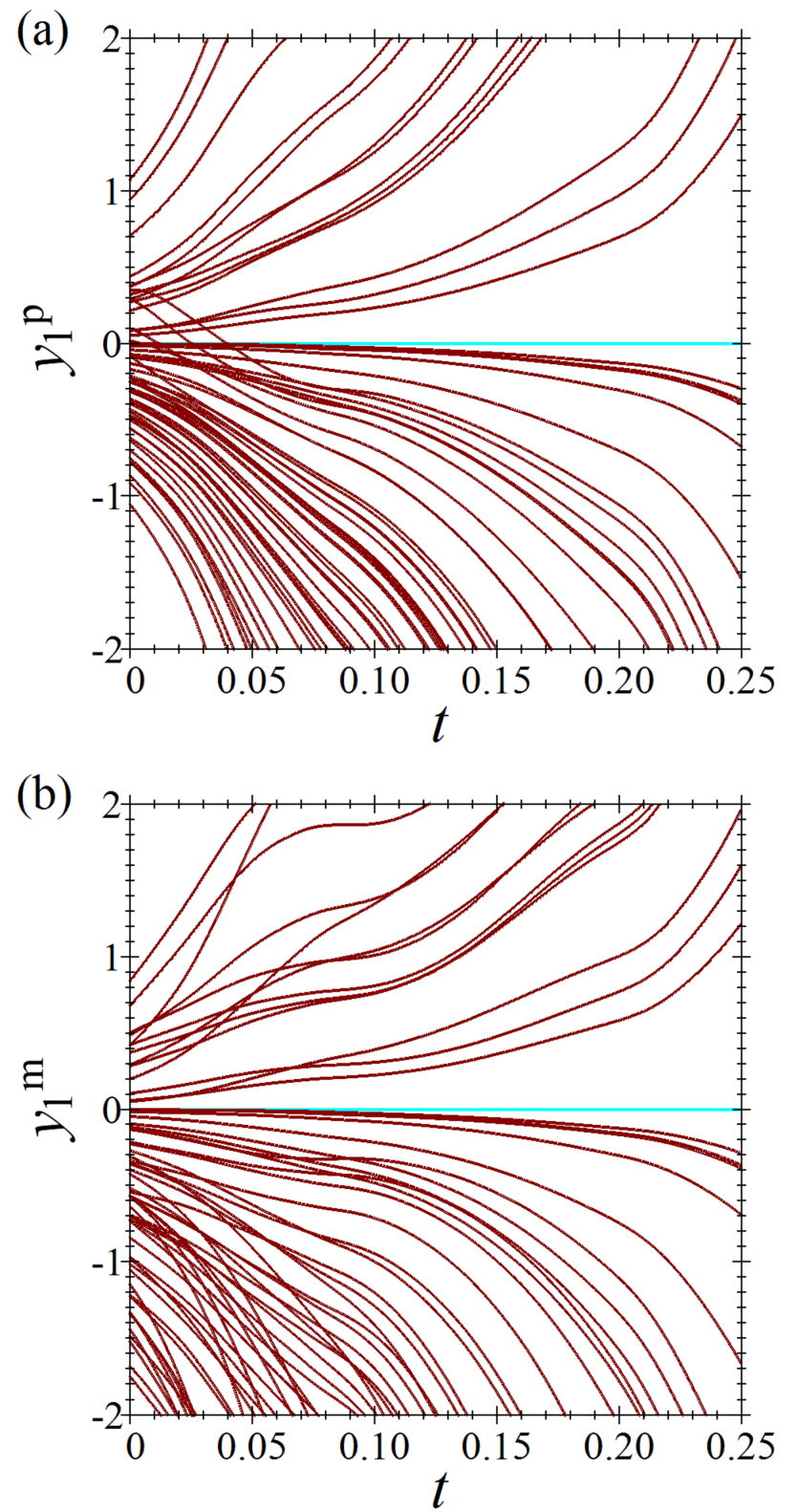

FIG. 7: Trajectories run with the temperature $k_{\mathrm{B}} T=4$, plotted in (a) time $t$ and the partial normal form coordinate $y_{1}^{\mathrm{p}}$, and (b) time $t$ and the minimal normal form coordinate $y_{1}^{\mathrm{m}}$. Light blue lines mark the set $y_{1}=0$.

mal NF can perform as a good approximation to the dynamics of the system. Figure 7 shows the plot of the trajectories at $k_{\mathrm{B}} T=4$ in (a) the $t-y_{1}^{\mathrm{p}}$ space, and (b) the $t-y_{1}^{\mathrm{m}}$ space, where $y_{1}^{\mathrm{m}}$ is obtained by the minimal NF (Sec. II A 2). In Fig. 7(a), we observe crossings between the trajectories and with the line $y_{1}^{\mathrm{p}}$, which would not be observed if the partial NF were a true description of the bluesystem. This shows that the partial NF does not describe the dynamics of the system correctly at this temperature (consistent with the error estimate in Fig. 4). We cannot use the set $y_{1}^{\mathrm{p}}=0$ as the reaction boundary at this temperature. In turn, the error estimate (Fig. 4) tells that the minimal NF can still give a good description of the dynamics at $k_{\mathrm{B}} T=4$. The trajectories are plotted in time $t$ and the minimal NF coordinate $y_{1}^{\mathrm{m}}$ in Fig. 7(b). We observe crossings between the trajectories and the phase space flow looks quite irregular in contrast to the clear lamination seen in Fig. 6 (b). This is because the minimal NF allows the couplings between $y_{1}^{\mathrm{m}}$ and the other coordinates [see Eq. (23)]. However, as discussed in Sec. II A 2, trajectories cannot cross the line $y_{1}^{\mathrm{m}}=0$, which then functions as the boundary of reaction. In this temperature region, the lamination structure in the domain $y_{1}^{\mathrm{m}} \neq 0$ is lost, whereas the invariant set $y_{1}^{\mathrm{m}}=0$ remains to exist.

At much higher temperature, even the minimal NF gives a poor description of the system (Fig. 4). We observed both crossing with the line $y_{1}^{\mathrm{m}}=0$ and crossings between the trajectories (not shown here), which results in the disappearance of well-defined reaction boundary as an analytical functional of variables to represent the system and the environment.

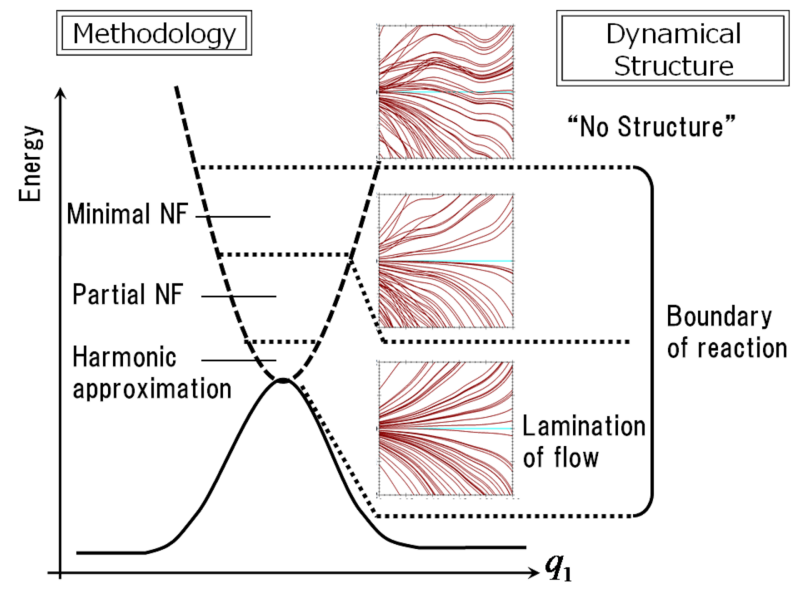

FIG. 8: A schematic picture of the hierarchical structure in the saddle region of condensed phase reaction dynamics. As the temperature increases, the system can have higher energy above the saddle point, causing the appropriate description of the true dynamics to change from the harmonic approximation to partial normal form, and then to minimal normal form. Very high temperature results in structureless dynamics. At the first two levels of the hierarchy, the dynamics along the reaction coordinate is decoupled from the rest degrees of freedom, and we can find lamination of the phase space flow. At the third level, the reaction coordinate ceases to be decoupled but yet the boundary of the reaction still survives.

\section{SUMMARY AND OUTLOOK}

Figure 8 shows schematically the hierarchical structure of crossing dynamics in the saddle region in the presence of stochastic, thermal fluctuation. At very low temperature where the system crossing the rank-one saddle experiences mainly the close proximity of a saddle point, the harmonic approximation is generally validated. As found in Refs. 6567 , at such low temperature, all the normal coordinates (including the reactive and the non-reactive ones) are decoupled from each other. The phase space flow is then laminated due to the absence of coupling. One can also find a clear reaction boundary classifying the system into reactive and non-reactive 
trajectories. As the temperature increases, the effect of nonlinearity of the potential and its nonlinear cooperation with the random force become significant. Nevertheless, up to moderately high temperature, it was shown by performing the partial $\mathrm{NF}$ transformation that one can extract a reaction coordinate decoupled from all the rest, with properly incorporating the effect of nonlinearity and environment, under thermal fluctuation. The phase space flow plotted as a function of the reaction coordinate and the time is essentially the same with the harmonic case. That is, there is a lamination of flow because of the absence of coupling and a clear reaction boundary given by the zero of the partial NF reaction coordinate.

As the temperature further increases, another level of hierarchy emerges at which the reaction coordinate is no longer decoupled as at lower temperature. In this temperature regime, the lamination structure is lost and the phase space looks irregular. However, by the method of the minimal NF we presented in this paper, the boundary which divides the system into the reactive and the nonreactive trajectories robustly persists with its analytical functional of the position coordinates and velocities of the system and friction constants and random force.

At very high temperature, the boundary starts to cease. That is, we cannot find any 'structure' in the reaction dynamics of the system to enable us to predict the final destination of the reaction. The reaction dynamics is expected to become purely stochastic and it may be inevitable to monitor all the paths of the system by many trajectory calculations for the determination of the reaction boundary. However, strictly speaking, it means that we cannot find any structure in the framework of NF which was formulated as a perturbation from the harmonic approximation. ${ }^{65-67}$ It does not completely exclude the possibility of emergence of new structures such as the change of stability of transition state due to the bifurcation. ${ }^{42,43,57}$

Note also that the boundaries between the different levels of the hierarchy shown in Fig. 8 are not exactly located at any clear place in the temperature or in the phase space. The errors of the harmonic, the partial and the minimal NF increase continuously with the temperature. There is no strict threshold when the approximation becomes "bad" and should be replaced by the the other, different level of approximation. The transition from one level to the next in Fig. 8 should be therefore understood as a gradual change, not as a sudden jump.

It is interesting to compare the hierarchical structure of transition states in Hamiltonian systems. ${ }^{11,40-43,56,57}$ One notable difference is that there exists no full normal form regime in condensed phase where all the degrees of freedom are decoupled from each other although it does in isolated gas phase. This is due to the fact that the random force generally has a wide range of frequency spectrum (especially, in the case of white noise, the power spectrum of the random force is uniform through all frequencies). Therefore, except the unstable reactive degree of freedom, all the nonreactive coordinates of the system should generally be subject to resonance with the surrounding environment in condensed phase. ${ }^{59,60}$ The other interesting subject is to investigate whether the corresponding minimal NF also exists for Hamiltonian systems, which has not been notified insofar. An equation similar to the minimal NF [Eq. (23)] should be able to be constructed in the framework of Hamiltonian systems. The minimal NF in the Hamiltonian systems may preserve the stable and the unstable manifolds as invariant sets, even if the action associated with reactive degree of freedom in the phase space does no longer preserve generically. We will present this subject in the forthcoming paper.

One of the most striking consequences of the reaction boundary in the phase space persisting up to moderately high temperature is that one can analytically calculate rate constants of chemical reactions without lying on the concept of transition state in condensed phase. Since the reactivity of given initial conditions in the region of saddle can be correctly assigned by using the present theory, we can calculate the reaction probability analytically. The reaction probability integrated through the Boltzmann distribution of initial conditions gives the rate of the reaction (under the assumptions of local equilibrium and of quantum effects being negligible). We are in progress in this subject. ${ }^{72}$

In this paper we have assumed a Langevin equation with the force-force autocorrelation given by the delta function. When the time scale of the solvent motion overlaps with that of the reacting system, the assumption starts to break down, and we need to introduce a generalized Langevin equation ${ }^{73}$ with the memory kernal proportional to the force-force autocorrelation function because of the fluctuation-dissipation theorem. The extension of the present framework of normal form will be presented in a separate paper. Our preliminary result indicates that the similar reaction boundary as the present work can also be found in the system obeying a generalized Langevin equation.

\section{ACKNOWLEDGMENTS}

This work has been supported by Research Fellowships of the Japan Society for the Promotion of Science for Young Scientists (to SK) and by JSPS, JST/CREST, Priority Area 'Molecular Theory for Real Systems' (to TK).
${ }^{1}$ R. D. Levine, Molecular Reaction Dynamics, Cambridge University Press, Cambridge, 2005.

2 H. Eyring, J. Chem. Phys., 1935, 3, 107-115.

3 E. Wigner, J. Chem. Phys., 1937, 5, 720-725.

4 M. G. Evans and M. Polanyi, Trans. Faraday Soc., 1935, 31, 875894.
5 O. K. Rice and H. C. Ramsperger, J. Am. Chem. Soc., 1928, 50, 617-620.

${ }^{6}$ L. S. Kassel, J. Phys. Chem., 1928, 32, 1065-1079.

7 R. A. Marcus, J. Chem. Phys., 1952, 20, 359-364.

8 J. C. Keck, Adv. Chem. Phys., 1967, 13, 85-121.

9 D. G. Truhlar and B. C. Garrett, Acc. Chem. Res., 1980, 13, 440- 
448

10 T. Uzer, C. Jaffé, J. Palacián, P. Yanguas and S. Wiggins, Nonlinearity, 2002, 15, 957-992.

11 T. Komatsuzaki and R. S. Berry, J. Chem. Phys., 1999, 110, 91609173.

12 C. Jaffe, S. D. Ross, M. W. Lo, J. Marsden, D. Farrelly and T. Uzer, Phys. Rev. Lett., 2002, 89, 011101_1-011101_4.

13 M. Karplus, J. Phys. Chem. B, 2000, 104, 11-27.

14 D. W. Oxtoby and S. A. Rice, J. Chem. Phys., 1976, 65, 1676.

15 M. J. Davis and S. K. Gray, J. Chem. Phys., 1986, 84, 5389.

16 H. A. Kramers, Physica, 1940, 7, 284-304.

17 R. F. Grote and J. T. Hynes, J. Chem. Phys., 1980, 73, 2715-2732.

18 A. M. Berezhkovskii, E. Pollak and V. Y. Zitserman, J. Chem. Phys., 1992, 97, 2422-2437.

19 E. Pollak and P. Talkner, Phys. Rev. E, 1993, 47, 922-933.

20 E. Pollak, A. M. Berezhkovskii and Z. Schuss, J. Chem. Phys., 1994, 100, 334-339.

21 G. van der Zwan and J. T. Hynes, J. Chem. Phys., 1983, 78, 41744185.

22 E. Pollak, Chem. Phys. Lett., 1986, 127, 178.

23 H. Mori, Prog. Theor. Phys., 1965, 33, 423-455.

24 R. Zwanzig, J. Stat. Phys., 1973, 9, 215-220.

25 M. Tokuyama and H. Mori, Prog. Theor. Phys., 1976, 55, 411429.

26 A. Nitzan, P. Ortoleva, J. Deutch and J. Ross, J. Chem. Phys., 1974, 61, 1056-1074.

27 T. Komatsuzaki and M. Nagaoka, J. Chem. Phys., 1996, 105, 10838.

28 T. Komatsuzaki and M. Nagaoka, Chem. Phys. Lett., 1997, 265, 91.

29 T. Komatsuzaki and R. S. Berry, Phys. Chem. Chem. Phys., 1999, 1, 1387.

30 T. Komatsuzaki and R. S. Berry, J. Chem. Phys., 2001, 115, 4105.

31 T. Komatsuzaki and R. S. Berry, J. Mol. Struct. (Theochem), 2000, 506, 55

32 T. Komatsuzaki and R. S. Berry, Proc. Nat. Acad. Sci. USA, 2001, 98, 7666 .

33 T. Komatsuzaki and R. S. Berry, J. Phys. Chem. A, 2002, 106, 10945.

34 S. Wiggins, L. Wiesenfeld, C. Jaffé and T. Uzer, Phys. Rev. Lett., 2001, 86, 5478-5481.

35 H. Waalkens, A. Burbanks and S. Wiggins, J. Chem. Phys., 2004, 121, 6207.

${ }^{36}$ H. Waalkens, A. Burbanks and S. Wiggins, J. Phys. A, 2004, 37, L257-L265.

37 H. Waalkens and S. Wiggins, J. Phys. A, 2004, 37, L435-L445.

38 S. Kawai, Y. Fujimura, O. Kajimoto, T. Yamashita, C.-B. Li, T. Komatsuzaki and M. Toda, Phys. Rev. A, 2007, 75, 022714.

39 S. Kawai, C. Jaffé and T. Uzer, J. Phys. B: At. Mol. Opt. Phys., 2005, 38, S261.

40 C.-B. Li, Y. Matsunaga, M. Toda and T. Komatsuzaki, J. Chem. Phys., 2005, 123, 184301.

41 C.-B. Li, A. Shojiguchi, M. Toda and T. Komatsuzaki, Few-Body
Systems, 2006, 38, 173.

42 C.-B. Li, A. Shojiguchi, M. Toda and T. Komatsuzaki, Phys. Rev. Lett., 2006, 97, 028302.

43 C.-B. Li, M. Toda and T. Komatsuzaki, J. Chem. Phys., 2009, 130, 124116.

44 E. R. Lovejoy, S. K. Kim and C. B. Moore, Science, 1992, 256, 1541-1544.

45 E. R. Lovejoy and C. B. Moore, J. Chem. Phys., 1993, 98, 78467854.

46 W. H. Miller, Faraday Discussions Chem. Soc., 1977, 62, 40-46.

47 T. Seideman and W. H. Miller, J. Chem. Phys., 1991, 95, 1768.

48 S. Keshavamurthy and W. H. Miller, Chem. Phys. Lett., 1993, 205, 96.

49 R. Hernandez and W. H. Miller, Chem. Phys. Lett., 1993, 214, 129-136.

50 R. Hernandez, J. Chem. Phys., 1994, 101, 9534.

51 R. A. Marcus, Science, 1992, 256, 1523-1524.

52 D. J. Wales and R. S. Berry, J. Phys. B, 1991, 24, L351.

53 R. J. Hinde, R. S. Berry and D. J. Wales, J. Chem. Phys., 1992, 96, 1376.

54 C. Amitrano and R. S. Berry, Phys. Rev. Lett., 1992, 68, 729-732.

55 R. J. Hinde and R. S. Berry, J. Chem. Phys., 1993, 99, 2942-2963.

56 T. Komatsuzaki and R. S. Berry, Adv. Chem. Phys., 2002, 123, 79.

57 S. Kawai, H. Teramoto, C.-B. Li, T. Komatsuzaki and M. Toda, Adv. Chem. Phys., 2009.

58 J. Moser, Comm. Pure Appl. Math., 1958, 11, 257.

59 S. Kawai and T. Komatsuzaki, J. Chem. Phys., 2009, 131, 224505.

60 S. Kawai and T. Komatsuzaki, J. Chem. Phys., 2009, 131, 224506.

61 A. Y. T. Leung and Q. C. Zhang, J. Sound Vib., 2003, 266, 261279.

62 S. Kawai, A. D. Bandrauk, C. Jaffé, T. Bartsch, J. Palacián and T. Uzer, J. Chem. Phys., 2007, 126, 164306_1-164306_12.

63 K. Müller and L. D. Brown, Theor. Chim. Acta, 1979, 53, 75-93.

64 D. L. Ermak and H. Buckholz, J. Comp. Phys., 1980, 35, 169182.

65 T. Bartsch, R. Hernandez and T. Uzer, Phys. Rev. Lett., 2005, 95, 058301_1-058301_4.

66 T. Bartsch, T. Uzer and R. Hernandez, J. Chem. Phys., 2005, 123, 204102_1-204102_14.

67 T. Bartsch, T. Uzer, J. M. Moix and R. Hernandez, J. Chem. Phys., 2006, 124, 244310_1-244310_13.

68 T. Bartsch, J. M. Moix, R. Hernandez, S. Kawai and T. Uzer, $A d v$. Chem. Phys., 2008, 140, 191-238.

69 A. Deprit, Cel. Mech., 1969, 1, 12.

70 A. Lichtenberg and M. Lieberman, Regular and Chaotic Dynamics, Springer, 1992.

71 W. H. Press, S. A. Teukolsky, W. T. Vetterling and B. P. Flannery, Numerical Recipes in C, Cambridge University Press, Cambridge, 1992.

72 S. Kawai and T. Komatsuzaki, J. Chem. Phys., inpreparation.

73 R. Zwanzig, Nonequilibrium Statistical Mechanics, Oxford University Press, London, 2001. 